\title{
Web Accessibility Requirements for Media Players
}

\author{
María González, Lourdes Moreno, Paloma Martínez, and Ana Iglesias \\ LaBDA Group, Computer Science Department, Universidad Carlos III de Madrid, \\ Avda. Universidad 30, 28911 Leganés, Madrid, Spain \\ \{mgonza1, lmoreno, pmf, aiglesia\}@inf.uc3m.es
}

\begin{abstract}
Video Content continues to strenghten on the Web, because of that fact, it is neccessary to include video content with suitable accessibility requirements to be used by all people. User Agent (such as player, browser), also have to include accessibility requirements. In this paper a set of basic guidelines is included for professionals who want to embed video players on their Web.
\end{abstract}

Keywords: Web accessibility, user agent, media player, standard, evaluation.

\section{Introduction}

The Web is being overflowed with multimedia content, like video. This will keep on happening into the future [1]. Not only multimedia content has to be accessible through standards like the Web Content Accessibility Guideline (WCAG) [1] of Web Accessibility Initiative (WAI) [3] among others, but it also has to satisfy accessibility requirements, which have independent components, for example those that are referred to the user agent such as media players, browser, etc.

Sometimes, accessibility problems appear because of accessibility barriers within the media player, such as browsers, assistive technologies or even user's inability, for example, when a user tries to watch a video, but do not have the appropriate software and do not how to install it.

To avoid these problems, media players have to be developed in agreement with User Agent Accessibility Guidelines (UAAG) [4] of WAI, as well as, having into account different opinions based on studies to offer universal access solutions for the user.

The remaining of the paper is organized as follows: Section 2 discusses different accessibility standards for media players and provides technological solutions. In Section 3, accessibility guidelines for media players are presented. Finally, Section 4 presents the concluding remarks and future works.

\section{Background}

In this section, standards for Web accessibility, regulations about how to standardize accessibility in software and good practices guidelines will be indicated, as well as new contributions that are provided by the new standard HTML5.

The UAAG of WAI is an international standard that explains how to make user agent accessible for people with disabilities and how to increase accessibility on Web 
content. Nowadays, this standard has two versions, UAAG 1.0 [5] (the reference version) and UAAG 2.0 [6] (the draft version). Last version gives support to WCAG 2.0 [7], being this one used to make the accessibility guidelines.

Another standard is ISO 9241-171:2008 [8], Ergonomics of human-system interaction, this standard provides a group of guidance on software accessibility.

The new standard HTML5 [9] allows to play videos without installing plug-ins through the new labels such as <video $>$ and <audio $>$. However, there are some problems due to the fact that it does not support all Web user agent completely in the keyboard access or through screen readers to the provided controls. On the other hand, the current draft version of HTML5 does not give support to include subtitles and audio description, so in order to become the official recommendation, it is necessary to include these requirements to satisfy UAAG 2.0 and WCAG 2.0 [10].

As well as these standards, there are works [11][12][13] and institutions [14][15][16] that provide good practices guidelines according to accessible multimedia content on the Web.

\section{Accessibility Guidelines for Web Media Players}

With the knowledge that we obtain after carrying out a study of WAI standards such as WCAG 2.0, the agile evaluation method based on UAAG 2.0 as well as the study of others standards and relative works, following, we present as result, a help documentation to guide web professional. The documentation includes a set of basic guidelines and a group of elements to be introduced on a media player interface so a video is accessible on a Web media player. This documentation is elaborated to help Web professionals.

1) A media player has to provide different alternatives for audiovisual information next to the video:

a. Captions (video subtitles). As it is shown in Figure 1.

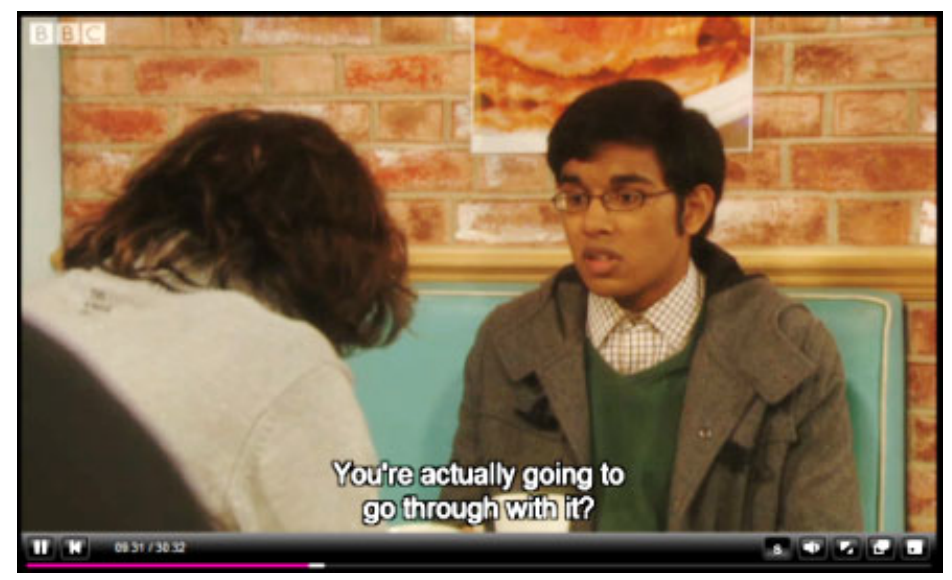

Fig. 1. BBC iPlayer's screenshot showing subtitles 
b. Audio description.

c. Others (sing language, transcription, extended audio description, etc.).

2) Complete access to all the features has to be guaranteed, as well as towards the mouse:

a. By keyboard.

b. Through assistive technology (like screen reader).

3) Provide help and documentation about the features of media player accessibility on the user interface, which reports to the user about the availability of those features, as well as the information about their purpose and use.

4) Provide a keyboard focus cursor that shows visually what element has the focus on the user interface; it is also important that the media player has a text cursor to show the focus within a text element. Restore the state when the state focus is recovered.

According to the previous guidelines, elements on the user interface have to be included like the following controls. These controls are divided in two groups: basic controls and additional controls that are necessary to obtain an accessible media player.

In the first group (basic controls) are included:

- Controls that allow users to play or to stop the video.

- Controls that allow users to resize the viewports.

- Controls that allow users to adjust the volume.

Among additional controls, we introduce the following ones:

- Controls that allow users to enable or to disable subtitles. As it is shown in Figure 2, there is a button called 'CC' (Closed Caption) that provides this functionality.

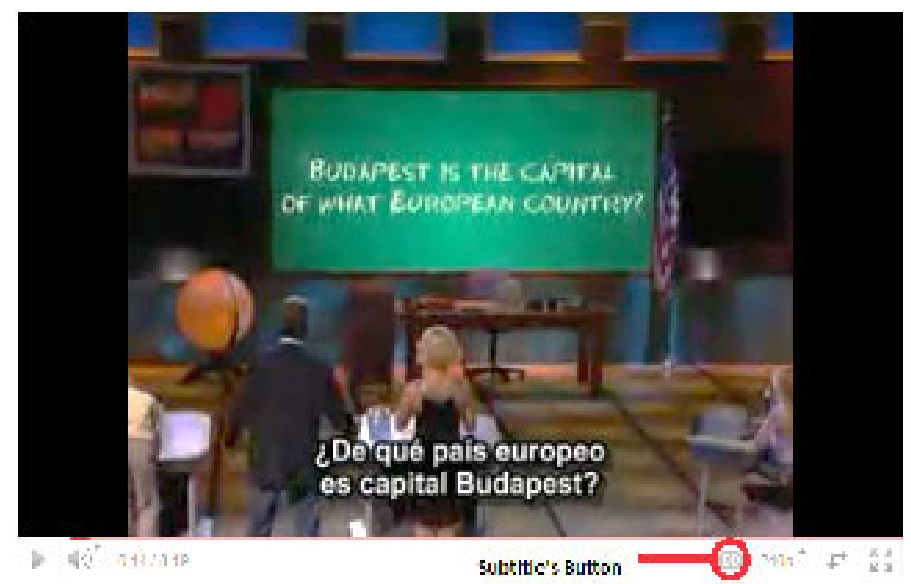

Fig. 2. YouTube's screenshot that shows $\mathrm{CC}$ button 
- Controls that allow users to enable or to disable the audio description.

- Controls that allow users to search within reproduction subtitles, as it is shown in Figure 3.

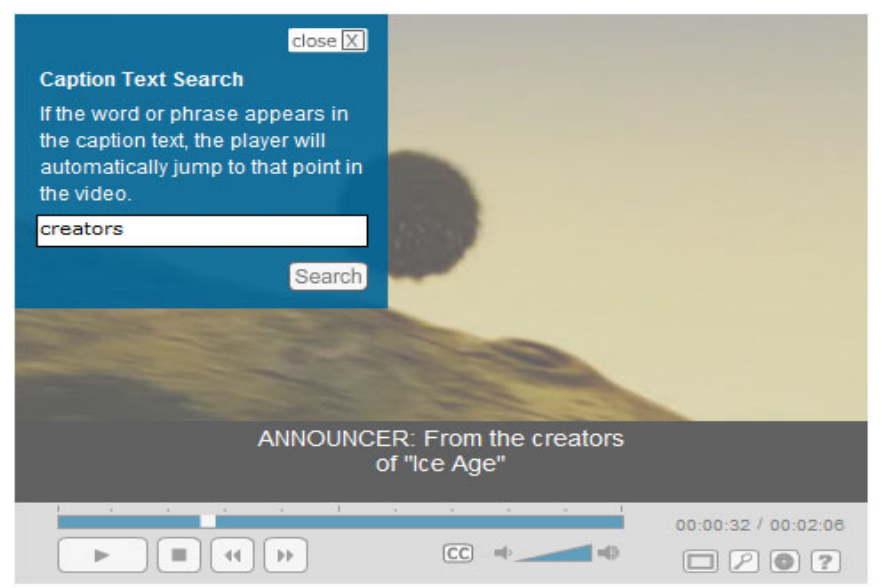

Fig. 3. CCplayer's screenshot that shows a caption search

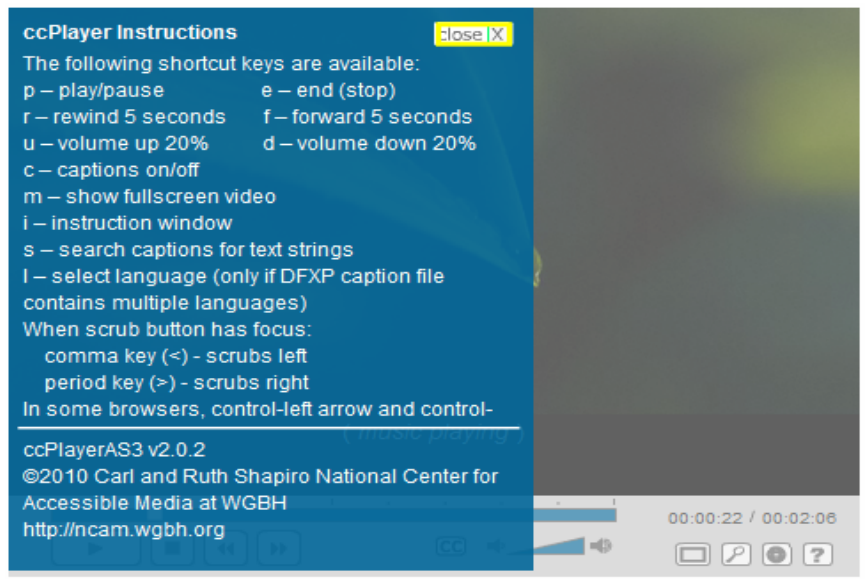

Fig. 4. CCPlayer's screenshot that shows a command's menu

- Controls that allow users to forward or to delay seconds within a reproduction.

- Controls that allow users to change the size, font or color of the text.

- Controls that allow users access to help documentation which reports keyboard shortcuts, as it is shown in Figure 4.

5) According to other important requirements concerning accessibility close to usability, the following requirements have been found: 
a) It is very important to maintain accessibility features that are configured by the users in the following sessions and that those features only change when the user want.

b) Controls that allow users to enable or to disable and to adjust accessibility features have to be easy to find and they have to be operable.

c) Provide information that allows the user to know the keyboard shortcuts that can be used in the media player, such as the help menu shows in Figure 4.

d) Allow navigating through the content without enabling any controls.

e) Has to be easy so the user can move through the menus, submenus and list through different combinations of keyboards or direct keyboard commands.

f) Allow users to set their preferences to configure the keyboard shortcuts.

\section{Conclusions}

Although Web multimedia content has grown, this growth is not comparable to the Web accessibility growth, because it is not accessible enough yet. Due to that fact, it is very important that the media players and their access through the Web are accessible.

This paper was elaborated to help Web professionals to create or to decide what media player has to be included on their Website to guarantee the same access for all and accessible content is provided. Because of this, a set of accessibility basic guidelines that have to be satisfied have been introduced to obtain an accessible media player. Besides, an agile method of evaluation is introduced, which was used previously to evaluate some media players, that help evaluators to prove if a media player labeled as accessible actually is.

Acknowledgments. This research work is supported by the Research Network MAVIR (S2009/TIC-1542 (see www.mavir.net/)), and GEMMA (TSI-020302-2010141) and SAGAS (TSI-020100-2010-184) research projects.

\section{References}

1. Cisco Visual Networking Index, http://www.cisco.com/en/US/solutions/collateral/ns341/ ns525/ns537/ns705/ns827/white_paper_c11481360_ns827_Networking_Solutions_White_Paper.html

2. W3C, WAI, Web Content Accessibility Guidelines, WCAG (2010), http: / / www . w3 . org/WAI / intro/wcag . php

3. W3C, Web Accessibility Initiative, WAI (2010), http://www.w3.org/WAI/

4. W3C, WAI, User Agent Accessibility Guidelines, UAAG (2010), http://www.w3.org/WAI/intro/uaag.php

5. W3C, User Agent Accessibility Guidelines 1.0 (2002), http: / / www.w3 . org/TR/WAI-USERAGENT/ 
6. W3C, User Agent Accessibility Guidelines 2.0 (2010), http://www.w3.org/TR/UAAG20/

7. W3C, Web Content Accessibility Guidelines 2.0 (2008), http: / / www .w3 . org/TR/WCAG20/

8. ISO, International Organization for Standardization (2008), http://www.iso.org

9. W3C, HTML5, A vocabulary and associated APIs for HTML and XHTML, http://www.w3.org/TR/2009/WD-html5-20090423/

10. Moreno, L., Martínez, P., Iglesias, A., González-García, M.: HTML5 support for an accessible user-video-interaction on the Web. In: 13th IFIP TC13 Conference on HumanComputer Interaction, INTERACT 2011, Lisbon, Portugal (September 2011) (in press)

11. WebAIM, Media Player Accessibility (2010), http://webaim.org/techniques/captions/mediaplayers/

12. Clark, J.: Accessibility, Design and Writing (2010), http: / / joeclark . org/

13. Moreno, L., Martínez, P., Ruiz-Mezcua, B.: Guías metodológicas para contenidos multimedia accesibles en la Web. In: X Congreso Internacional de Interacción PersonaOrdenador, Interacción 2009, Barcelona, España (September 2009) ISBN: 13:978-84-692

14. Alfred, P.: Sloan Foundation (2008), http: / / www.sloan. org/

15. NCAM, National Center for Accessible Media (2009), http: / / ncam.wgbh. org/

16. Stanford Captioning, http://captioning.stanford.edu/ 